\title{
Extrapulmonary lymphomatoid granulomatosis presenting as Pancoast's syndrome
}

\author{
G. Dolan, J. Smith and J.T. Reilly \\ Departments of Haematology and ${ }^{1}$ Histopathology, Northern General Hospital, Herries Road, Sheffield \\ $S 77 A U, U K$
}

\begin{abstract}
Summary: We report the case of a 55 year old man who presented as Pancoast's syndrome associated with a left sided Horner's syndrome, a mass in the left supraclavicular fossa and marked weakness with wasting of the left arm consistent with a left sided brachial plexus lesion. Biopsy revealed the typical features of lymphomatoid granulomatosis. Neurological involvement is a rare presenting manifestation of this uncommon disease. Lymphomatoid granulomatosis should be considered as a rare but potentially treatable cause of Pancoast's syndrome.
\end{abstract}

\section{Introduction}

Lymphomatoid granulomatosis is a disease characterized by an angiocentric and angiodestructive atypical lymphocytoid and plasmacytoid infiltrate of various organs. The infiltrate and associated vasculitis can lead to the gross appearance of caseating granulomata. ${ }^{1}$ There has been much debate about the nature and classification of this disorder but it is probably now best considered as a peripheral $\mathrm{T}$ cell lymphoma. ${ }^{2}$ Pulmonary involvement is the most common clinical manifestation and although neurological manifestation is well recognized, it is the presenting feature in only a minority of cases. ${ }^{3}$ This case demonstrates that this rare disease should be added to the list of causes of Pancoast's syndrome.

\section{Case report}

A 55 year old man presented with a 6-week history of progressive weakness of his left arm associated with a mass on his left chest wall. He smoked 20 cigarettes daily and had lost $7 \mathrm{~kg}$ in weight. On examination he appeared thin and unwell, with a diffuse soft tissue mass extending from the left axilla to the left supraclavicular fossa. There was flaccid paralysis of the left arm with neurological signs, consistent with a lower brachial plexus lesion, and marked lymphoedema. He had a left Horner's syndrome and moderate hepatomegaly.

Correspondence: G. Dolan, M.B., Ch.B., M.R.C.P., M.R.C.Path.

Accepted: 4 March 1991
A provisional diagnosis of metastatic bronchial carcinoma was made.

Investigations revealed that he had opacification at the apex of the left lung, soft tissue swelling in the supraclavicular fossa and elevation of the left hemidiaphragm. The erythrocyte sedimentation rate was $25 \mathrm{~mm}$ in the first hour, blood count, urea and electrolytes were normal. Serum alkaline phosphatase was raised at $503 \mathrm{IU} / 1$ and serum transaminases were normal. Biopsy of the mass showed an atypical lymphocytoid and plasmacytoid, angiocentric and angiodestructive infiltrate with direct involvement of neural tissue (Figure 1). The atypical cells showed positive immunohistochemical staining with the T-cell marker UCH L1 and negative staining with the B-cell marker L26 (Dako Ltd). A diagnosis of lymphomatoid granulomatosis was made. Computerized tomography demonstrated multiple pulmonary nodules and widespread lymphodenopathy. He was treated with CHOP chemotherapy and after two courses, significant reduction in the size of the superficial mass occurred. Shortly after his second course he had a sudden, massive gastrointestinal haemorrhage and died. Permission for post-mortem examination was refused.

\section{Discussion}

Pulmonary disease usually dominates the clinical course of lymphomatoid granulomatosis and, even when not clinically manifest, it is almost invariably found during investigation, as in this case. Central nervous system involvement has been reported to occur in up to $30 \%$ of cases. To our knowledge 


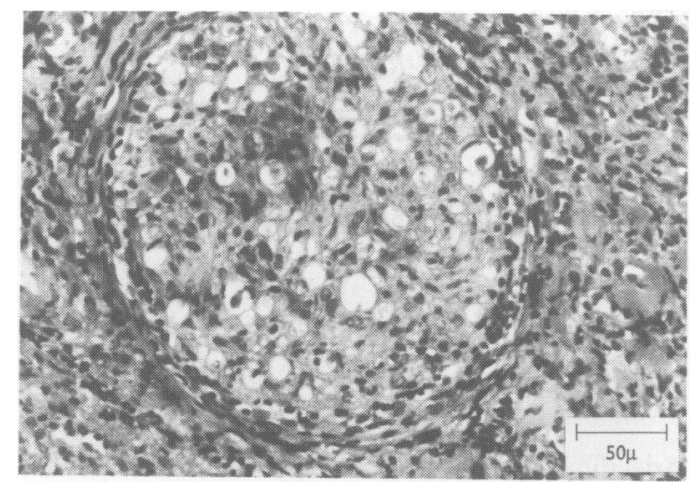

Figure 1 Photomicrograph showing infiltration of a peripheral nerve by atypical lymphoid cells resulting in neuronal vacuolation and degeneration. Haematoxylin \& eosin, original magnification $\times 65$.

there have been no reports of a Pancoast syndrome. ${ }^{3-5}$ Lymphomatoid granulomatosis is rare and as there has been controversy over its classification, there has been difficulty assessing the

\section{References}

1. Liebow, A.A., Carrington, C.R.B. \& Friedman, P.J. Lymphomatoid granulomatosis. Hum Pathol 1972, 3: 457-558.

2. Gallagher, C.J. Rare tumours: peripheral T cell lymphoma. Cancer Topics 1990, 7: 123-125.

3. Fauci, A.S., Haynes, B.F., Costa, J. Katz, P. \& Wolff, S.M. Lymphomatoid granulomatosis. Prospective clinical and therapeutic experience over 10 years. $N$ Engl J Med 1982, 306: $68-74$. natural history, prognosis and response to treatment. The experience of earlier authors suggested that the disease carried a very poor prognosis. ${ }^{3}$ Death was reported in most cases as being due either to pulmonary disease or transformation to aggressive lymphoma which occurred in up to $47 \%$ of patients. ${ }^{1-3}$ The high mortality rate in earlier series may in part have been due to a reluctance to treat with chemotherapy as the disease was not uniformly accepted as maligant. Several authors were, however, successful in achieving lasting remissions with chemotherapy and some have suggested that prevention of the development of high grade lymphoma occurs as a result of early treatment. ${ }^{3}$ Our patient responded initially to chemotherapy consisting of cyclophosphamide, vincristine, daunorubicin and prednisolone (CHOP regime). Although no autopsy was performed, we believe his death was not directly due to his presenting disease but was an acute complication of treatment. This case demonstrates that biopsy of Pancoast lesions is essential, as rare, but treatable, causes may be identified.

4. Katzsenstein, A.A., Carrington, C.B. \& Liebow, A.A. Lymphomatoid granulomatosis: a clinicopathological study of 152 cases. Cancer, 43: 360-373.

5. Hogan, P.J., Greenberg, M.K. \& McCarty, G.E. Neurological complications of lymphomatoid granulomatosis. Neurology 1981, 31: 619-620. 